Pacific Journal of Mathematics

ALGEBRAS OF BOUNDED SEQUENCES

ALGT MYron HOFfman AND AR LAN BRUCE RAMSAY 


\title{
ALGEBRAS OF BOUNDED SEQUENCES
}

\author{
Kenneth Hoffman and Arlan Ramsay
}

Let $l^{\infty}$ be the algebra of all bounded sequences of complex numbers. The primary purpose of this paper is to settle, by means of a counter-example, a conjecture about subalgebras of $l^{\infty}$ : If $A$ is a subalgebra of $l^{\infty}$ which is closed under uniform convergence and separates the points of $\beta N$, then $A=l^{\infty}$.

The main tool used in the construction of the example is a positive result about Boolean algebras (Proposition 1), which seems interesting in its own right.

Our interest in subalgebras of $l^{\infty}$ stems from recent work on interpolation problems. In 1957, R. C. Buck inquired as to which sequences $\left\{z_{n}\right\}$ in the unit disc of the plane have this property: If $\left\{w_{n}\right\}$ is an arbitrary bounded sequence of complex sequence of complex numbers, there exists a function $f$, bounded and analytic in the unit disc, such that $f\left(z_{n}\right)=w_{n}$ for each $n$. This question was answered very effectively by L. Carleson [2]. It was also answered in a slightly weaker form by D. J. Newman [7]; and, it was partially answered by W. Hayman [5].

Badé and Curtis [1] have obtained strong results on subalgebras of $C(X)$, the algebra of continuous complexvalued functions on a compact Hausdorff space $X$, in case $X$ has the property that any two disjoint open $F_{\sigma}$ subsets have disjoint closures. The smallest nonfinite space with that property is $\beta N$, the Čech compactification of the integers. Since $l^{\infty}$ is isomorphic to $C(\beta N)$, the work of Badé and Curtis has shed some light on a general class of interpolation problems of the type which we previously mentioned [see 6; page 205].

Technical aspects of the work of Badé and Curtis, as well as similar aspects of the interpolation problem for bounded analytic functions, have led to the aforementioned conjecture. Were this conjecture true, it would be a powerful tool in the study of interpolation problems.

We shall settle the conjecture in the negative. We show that, if the continuum hypothesis is valid, there exists a subalgebra $A$ of $l^{\infty}$ such that

(i) $A$ is closed under uniform convergence;

(ii) every nonnegative sequence in $l^{\infty}$ is the modulus of a sequence in $A$;

(iii) $A \neq l^{\infty}$.

The example is constructed as follows. Let $\mathscr{B}$ be the Boolean

Received August 27, 1964. 
algebra of measurable subsets of the unit circle, modulo null sets. Let $B$ be the Boolean algebra of subsets of the integers modulo finite sets. As a lattice, $B$ has an unusual property (property (ii) of Lemma 4). We exploit that property and the continuum hypothesis, to produce an isomorphism of $\mathscr{B}$ into $B$. We extend the inverse of the isomorphism to a homomorphism from $B$ onto $\mathscr{B}$. The adjoint of such a homomorphism is a homeomorphism of the Stone space $S(\mathscr{B})$ into the Stone space $S(B)$. The space $S(\mathscr{B})$ is the maximal ideal space of $L^{\infty}$, the Banach algebra of essentially bounded measurable functions on the unit circle. The space $S(B)$ is $\beta N-N$. From the algebra of bounded analytic functions in the unit disc, we construct a subalgebra of $L^{\infty}=C(S(\mathscr{B}))$, and carry that algebra with the imbedding of $S(\mathscr{B})$ in $\beta N-N$, to obtain the desired subalgebra of $l^{\infty}$.

Homomorphisms of Boolean algebras. In a Boolean algebra $B$, denote least upper bounds by $\vee$, greatest lower bounds by $\wedge$, and complements by '. Denote the corresponding set operations by $\cup, \cap$, and '. If $a \leqq b$, let $[a, b]$ be the set of all $x$ in $B$ such that $a \leqq x \leqq b$. If $E$ is a subalgebra of $B$ and $x$ is an element of $B$, let $E[x]$ be the subalgebra generated by $E$ and $x$ :

$$
E[x]=\left\{(e \wedge x) \vee\left(f \wedge x^{\prime}\right) ; e, f \in E\right\} .
$$

We shall be concerned with the problem of extending Boolean algebra homomorphisms. The basic extension lemma is the following, implicitly given by Sikorski in [8].

LEMMA 1. Let $E$ be a subalgebra of the Boolean algebra $B$, and let $\phi$ be a homomorphism of $E$ into a Boolean algebra $C$. If $x \in B$, then $\varphi$ can be extended to a homomorphism of $E[x]$ into $C$ if and only if there exists an element $y$ in $C$ such that

(i) $\varphi([0, x] \cap E) \subseteq[0, y] \cap \varphi(E)$

(ii) $\varphi([x, 1] \cap E) \leqq[y, 1] \cap \varphi(E)$.

For each such $y$, there is a unique extension $\varphi^{+}$which takes $x$ into $y$; and then $\varphi^{+}(E[x])=\varphi(E)[y]$. If $\varphi$ is an imbedding and $y$ is such that equality holds in (i) and (ii), then the corresponding extension $\varphi^{+}$is also an imbedding.

Proof. We content ourselves with the remark that, if $y$ satisfies (i) and (ii), the extension $\varphi^{+}$is given by

$$
\varphi^{+}\left(\left(e_{1} \wedge x\right) \vee\left(e_{2} \wedge x^{\prime}\right)\right)=\left(\varphi\left(e_{1}\right) \wedge y\right) \vee\left(\varphi\left(e_{2}\right) \wedge y^{\prime}\right) \text {. }
$$

Lemma 2. (Sikorski) If $B$ is a Boolean algebra and $\varphi$ is a homomorphism of a subalgebra of $B$ into a complete Boolean algebra $C$, then $\phi$ can be extended to a homomorphism of $B$ into $C$. 
Proof. In the context of Lemma 1, we have

$$
\sup \varphi([0, x] \cap E) \leqq \inf \varphi([x, 1) \cap E) .
$$

Therefore, the element $y$ can always be found. Apply Zorn's lemma.

Of course, Lemma 2 says that complete Boolean algebras are injective in the category of Boolean algebras and Boolean homomorphisms.

An $F$-space is a topological space in which any two disjoint open $F_{\sigma}$ subsets have disjoint closures. A Boolean algebra is an F-algebra if its Stone space is an $F$-space. An $F$-algebra can also be described as one which satisfies property (I) of Badé and Curtis [1]: If $x_{1}, y_{1}, x_{2}, y_{2}, \cdots$ is an orthogonal sequence in $B$, then there exist orthogonal elements $x$ and $y$ in $B$, such that $x$ is an upper bound of $\left\{x_{1}, x_{2}, \cdots\right\}$ and $y$ is an upper bound of $\left\{y_{1}, y_{2}, \cdots\right\}$.

LemMa 3. A homomorphic image of an $F$-algebra is an F-algebra. If $B$ is an F-algebra in which orthogonal sets are (at most) countable, then $B$ is complete.

Proof. Let $B$ and $C$ be Boolean algebras, and let $\varphi$ be a homomorphism from $B$ onto $C$. If $c_{1}, c_{2}, \cdots$ is an orthogonal sequence in $C$, there can be defined (by induction) an orthogonal sequence of pre-images in $B$. Choose any $b_{1}$ such that $\varphi\left(b_{1}\right)=c_{1}$. If $b_{1}, \cdots, b_{k}$ are orthogonal with $\varphi\left(b_{j}\right)=c_{j}, 1 \leqq j \leqq k$, choose any $x$ in $B$ with $\varphi(x)=c_{k+1}$ and set $b_{k+1}=\left(b_{1} \vee \cdots \vee b_{k}\right)^{\prime} \wedge x$. From the alternative description of $F$-algebras, it is then clear that $C$ is an $F$-algebra if $B$ is.

Now, suppose that $B$ is an $F$-algebra and that orthogonal sets in $B$ are at most countable. To prove that $B$ is complete, it suffices to show that every orthogonal set in $B$ has an upper bound. Evidently, the only case of interest is that of an orthogonal sequence $x_{1}, x_{2}, \ldots$ which is part of a maximal orthogonal set $\left\{x_{1}, y_{1}, x_{2}, y_{2}, \cdots\right\}$. Since $B$ is an $F$-algebra, there exist orthogonal elements $x, y$ in $B$ such that $x$ is an upper bound of $\left\{x_{1}, x_{2}, \cdots\right\}$ and $y$ is an upper bound of $\left\{y_{1}, y_{2}, \cdots\right\}$. Then $x$ must be the least upper bound of $\left\{x_{1}, x_{2}, \cdots\right\}$. For, if $u$ is another upper bound of $\left\{x_{1}, x_{2}, \cdots\right\}$, then $z=x \wedge u$ is also such an upper bound. If $z$ were less than $x$, we could adjoin $z^{\prime} \wedge x$ to $\left\{x_{1}, y_{1}, x_{2}, y_{2}, \cdots\right\}$ and obtain a properly larger orthogonal set.

Examples of $F$-algebras abound. Evidently, any Boolean $\sigma$-algebra is an $F$-algebra. Consequently, any homomorphic image of a $\sigma$-algebra is an $F$-algebra. In particular, if $K$ is a closed subset of the Stone space of a $\sigma$-algebra, then the algebra of all (relatively) open-closed subsets of $K$ is an $F$-algebra. Of course, the same is true of any closed subset of the Stone space of an $F$-algebra. In other words, a closed subset of a (compact and totally disconnected) $F$-space is an $F$-space. 
One can use Lemma 3 to show that certain homomorphic images of $\sigma$-algebras are complete. We are interested in some homomorphic images of $\sigma$-algebras which might be called anti-complete, because, they contain no strictly increasing sequence which has a least upper bound. One such algebra is the algebra of subsets of the integers modulo finite sets, i.e., the algebra of open-closed subsets of the space $\beta N-N$. The following lemma provides us with a class of algebras of the type which we have in mind.

Lemma 4. Let $\mathscr{B}$ be an infinite Boolean $\sigma$-algebra, with Stone space $S$. Let $K$ be a nonempty closed $G_{\delta}$ in $S$ such that the interior of $K$ is empty. Let $B$ be the Boolean algebra of (relatively) open-closed subsets of $K$.

(i) Each maximal orthogonal set in $B$ is either finite or uncountable.

(ii) If $a_{1} \leqq a_{2} \leqq \cdots$ and $b_{1} \geqq b_{2} \geqq \cdots$ in $B$, and if $a_{n}<b_{n}$ for every $n$, then there is an element $c$ in $B$ such that $a_{n}<c<b_{n}$ for every $n$.

Proof. If $x$ is in $\mathscr{B}$, let $[x]$ be the corresponding open-closed subset of the Stone space $S$. The fact that $K$ is a nonempty closed $G_{\delta}$ without interior means that

$$
K=\bigcap_{n}\left[e_{n}\right]
$$

where $e_{1}>e_{2}>\cdots$ is a strictly decreasing sequence in $\mathscr{B}$ and $\inf _{n} e_{n}=$ 0 . The map

$$
\gamma(x)=[x] \cap K
$$

is a homomorphism from $\mathscr{B}$ onto $B$, and, $\gamma(x)=\gamma(y)$ if and only if there exists an $n$ such that $x \wedge e_{n}=y \wedge e_{n}$.

For the proof of (i), suppose that $\left\{a_{1}, a_{2}, \cdots\right\}$ is a countable orthogonal set in $B$. We shall show that there exists a nonzero element of $B$ which is orthogonal to each $a_{n}$. We may assume that each $a_{n}$ is nonzero. Let $x_{1}, x_{2}, \cdots$ be an orthogonal sequence in $B$ with $\gamma\left(x_{n}\right)=a_{n}$. If $k$ is a positive integer, then $\gamma\left(x_{k}\right)=a_{k} \neq 0$; hence, $x_{k} \wedge e_{n} \neq 0$ for all $n$. Thus, we must have $x_{k} \wedge\left(e_{n}-e_{n+1}\right) \neq 0$ for infinitely many values of $n$. From this it is clear that there is a sequence of integers $n_{1}<n_{2}<\cdots$ such that the elements

$$
y_{k}=x_{k} \wedge\left(e_{n_{k}}-e_{n_{k}+1}\right)
$$

are nonzero, $k=1,2, \cdots$. Let $y=\sup _{k} y_{k}$. For each $k$, the element $y \wedge e_{n_{k}+1}$ is orthogonal to $x_{k}$. Therefore, $\gamma(y)$ is orthogonal to each $a_{k}$. Since 


$$
y \wedge e_{k} \geqq y \wedge e_{n_{k}} \geqq y_{k}>0,
$$

we see that $\gamma(y) \neq 0$.

The proof of (ii) is essentially the same as the proof that every nonempty closed $G_{\delta}$ in the space $\beta N-N$ has a nonempty interior [4; pages 98,99$]$. First note that $B$ has no atoms. Such an atom would correspond to a point of $K$ which is isolated in $K$. The point would then be a $G_{\delta}$ in $S$. Any such point is isolated in $S$ and must be in the interior of $K$. Since $B$ has no atoms, (ii) is trivial if both sequences are eventually constant. Otherwise, the sequence $a_{1}, b_{1}, a_{2}-a_{1}, b_{1}-b_{2}, \cdots$ has infinitely many nonzero terms. By (i), there exist nonzero elements $a, b$ in $B$ such that the sequence $a, b, a_{1}, b_{1}^{\prime}, a_{2}-a_{1}, b_{1}-b_{2}, \cdots$ is orthogonal. Since $B$ is an $F$-algebra, there is an element $c$ in $B$ which is $\left\{b, b_{1}^{\prime}, b_{1}-b_{2}, \cdots\right\}$ and which is an upper bound of $\left\{a, a_{1}, a_{2}-a_{1}, \cdots\right\}$. Then, for each $n$

$$
a_{n}<a_{n} \vee a \leqq c \leqq b_{n}-b<b_{n} .
$$

That completes the proof.

We now focus our attention on a Boolean algebra $B$, which has property (ii) of Lemma 4. As we have indicated, the algebra of openclosed subsets of $\beta N-N$ is one such algebra. It is easy to see that a Boolean algebra $B$ has property (ii) if and only if its Stone space $S(B)$ has these three topological properties:

(a) $S(B)$ is an $F$-space;

(b) every nonempty (closed) $G_{\delta}$ in $S(B)$ has a nonempty interior;

(c) $S(B)$ has no isolated points.

We might remark that, if $S(B)$ satisfies (a), (b), and (c), then the Stone space for the algebra of Borel sets in $S(B)$ modulo first category sets is a Stonian space in which every first category set is nowhere dense and on which every measure has nowhere dense support. See Dixmier [3].

Lemma 5. Let $B$ be a Boolean algebra which has property (ii) of Lemma 4. Let $I$ be a countably generated ideal in $B$, and let $F$ be a countable subset of $B$ which is disjoint from $I$. If $y$ is a strict upper bound of $I$, there exists an element $z$ in $B$ such that

(a) $z<y$

(b) $z$ is a strict upper bound of $I$

(c) $F \cap[0, z]$ is empty.

Proof. First let us show that, if $u$ is an element of $B$ which is not in $I$, there is an $x \leqq y$ such that $u \not \equiv x$ and $x$ is a strict upper bound of $I$. We may suppose that $u \leqq y$; otherwise we may take $x=y$. Since $u$ is not in $I$, the ideal generated by $I$ and $u^{\prime}$ is proper. Let $J$ be that ideal. Since $J$ is countably generated, we can find $t<1$ such 
that $t$ is a strict upper bound of $J$. Choose $t_{1}$ with $0<t_{1}<t^{\prime}$, and let $x=y-t_{1}$. It is easy to see that $x$ is a strict upper bound of $I$ and that $u \geqq x$.

If $F=\left\{f_{1}, f_{2}, \cdots\right\}$, the preceding paragraph shows us how to construct a sequence $y_{1} \geqq y_{2} \geqq \cdots$ such that each $y_{n}$ is a strict upper bound of $I$ and such that, for each $n$, we have $f_{n} \not y_{n}$. Since $B$ has property (ii) of Lemma 4 , there exists an element $z$ in $B$ which is a strict upper bound of $I$ and is a strict lower bound of the dual ideal generated by $y_{1}, y_{2}, \cdots$. Any such $z$ has properties (a), (b), and (c).

Proposition 1. Let $B$ be a Boolean algebra which has property (ii) of Lemma 4. Any Boolean algebra of cardinality at most $\aleph_{1}$ can be imbedded in $B$.

Proof. Let $C$ be a Boolean algebra, the cardinality of which does not exceed $\aleph_{1}$. Then $C$ is the union of a well-ordered chain of countable subalgebras. We can arrange that the chain starts with the subalgebra $\{0,1\}$ and that each member of the chain is generated by the preceding subalgebras and one additional element. By Lemma 1 and induction, it suffices to prove the following. If $E$ is a countable subalgebra of $C$, if $\varphi$ is an isomorphism of $E$ onto the subalgebra $F$ of $B$, and if $x$ is in $C-E$, then there exists an element $y$ in $B$ such that

(a) $\phi([0, x] \cap E)=[0, y] \cap F$

(b) $\varphi([x, 1] \cap E)=[y, 1] \cap F$.

Let $I$ be the ideal in $B$ which is generated by the left-hand member of (a), and let $D$ be the dual ideal in $B$ which is generated by the left-hand member of (b). Then, both $I$ and $D$ are countably generated, and each element of $D$ is a strict upper bound of $I$. If we apply property (ii) of Lemma 4 and then Lemma 5 , we obtain an element $u_{0}$ of $B$ which is a strict upper bound of $I$ and is a strict lower bound of $D$, while $\left[0, u_{0}\right]$ and $F-I$ are disjoint.

Now, we show that, if $v$ is any element of $F-D$, then there exists $u$ such that $u \geqq u_{0} ; v \geqq u ; u$ is a strict lower bound of $D$; and $[0, u] \cap(F-I)$ is empty. If $v \supsetneqq u_{0}$, we may take $u=u_{0}$. If $v \geqq u_{0}$, observe that the dual ideal $D_{1}$, which is generated by $D \cup\left\{v^{\prime}\right\}$, is both countably generated and proper. Hence, there exists a nonzero element $w$ in $B$ which is a strict lower bound of $D_{1}$. Then $0<w<v^{\prime}$, and $u_{0} \vee w$ is a lower bound of $D$, with $u_{0}<u_{0} \vee w$. We apply Lemma 5 to the ideal $\left[0, u_{0}\right]$ and the subset $F-I$. We obtain an element $u$ such that $u_{0}<u<u_{0} \vee w$ and $(F-I) \cap[0, u]$ is empty. Then $u$ is a strict lower bound of $D$, and $v \geqq u$, because $u \wedge v^{\prime} \geqq u \wedge w>0$.

Suppose $F-D=\left\{v_{1}, v_{2}, \cdots\right\}$. We employ the argument which we have just concluded, to construct an increasing sequence $u_{0} \leqq u_{1} \leqq u_{2} \leqq \cdots$ such that, for each $n$, we have the following: $v_{n} \geqq u_{n} ;\left[0, u_{n}\right] \cap(F-I)$ 
is empty; $u_{n}$ is a strict lower bound of $D$. Once again we apply property (ii) of Lemma 4 and then Lemma 5, to conclude that there exists an element $y$ in $B$ such that $[0, y] \cap(F-I)$ is empty, $y$ is a strict lower bound of $D$, and $y$ is a strict upper bound of the ideal generated by $u_{1}, u_{2}, \cdots$. This is the $y$ which we seek.

Corollary. Let $B$ be an infinite Boolean F-algebra. Let $C$ be a Boolean algebra of cardinality at most $\aleph_{1}$ in which orthogonal sets are at most countable. If $C^{+}$is the completion of $C$ by cuts, then $C^{+}$is a homomorphic image of $B$. Hence, the Stone space $S\left(C^{+}\right)$can be imbedded in the Stone space $S(B)$.

Proof. Select any (infinite) discrete sequence in the Stone space $S(B)$. Its closure is homeomorphic to the space $\beta N$. Hence, the algebra of open-closed subsets on $\beta N-N$ is a homomorphic image of $B$.

We see that we need only prove the Corollary when $B$ is the Boolean algebra of open-closed subsets of the space $\beta N-N$. In that case, Proposition 1 provides us with an isomorphism $\varphi$, from a subalgebra of $B$ onto the algebra $C$. We can extend $\varphi$ to a homomorphism $\psi$, from $B$ into the completion $C^{+}$. Since orthogonal sets in $C$ are at most countable, the same is true in the algebra $C^{+}$. By Lemma 3, the range of the homomorphism $\psi$ is complete. Hence, $\psi$ maps $B$ onto $C^{+}$.

Suppose $C_{0}$ and $C_{1}$ are the free Boolean algebras on $\boldsymbol{\aleph}_{0}$ and $\boldsymbol{\aleph}_{1}$ generators, respectively. Each of these algebras satisfies the conditions on the algebra $C$ in the Corollary. Therefore, the Stone spaces $S\left(C_{0}^{+}\right)$ and $S\left(C_{1}^{+}\right)$can be imbedded in $\beta N-N$. In particular, $\beta N-N$ contains closed subsets which are Stonian but not hyper-Stonian [3].

Proposition 2. Assume the continuum hypothesis. Let $B$ be the Boolean algebra of subsets of the integers, modulo finite sets. If $\mathrm{C}$ is a complete Boolean algebra of cardinality at most $2^{\boldsymbol{N}_{0}}$, then $C$ is a homomorphic image of $B$. Hence, the Stone space $S(C)$ can be imbedded in the space $\beta N-N$.

Proof. Since $2^{\aleph_{0}}=\boldsymbol{\aleph}_{1}$, Proposition 1 tells us that $C$ can be imbedded in $B$. Then, we have an isomorphism $\varphi$, from a subalgebra of $B$ onto the algebra $C$. Since $C$ is complete, $\varphi$ can be extended to a homomorphism of $B$ onto $C$.

The example. Let $L^{\infty}$ be the algebra of essentially bounded measurable functions on the unit circle in the plane. Identify functions which agree almost everywhere. Then, with the essential supremum as norm, $L^{\infty}$ is a commutative Banach algebra which is isometrically isomorphic 
to $C(X)$, the algebra of continuous functions on its maximal ideal space $X$. The isomorphism is the Gelfand representation $f \rightarrow \widehat{f}$. We let $H^{\infty}$ be the subalgebra of $L^{\infty}$ which consists of the functions $h$ whose negative Fourier coefficients vanish. If $h \in H^{\infty}$ then the Poisson formula

$$
h(z)=\frac{1}{2 \pi} \int_{-\pi}^{\pi} h\left(e^{i \theta}\right) R e \frac{e^{i \theta}+z}{e^{i \theta}-z} d \theta
$$

extends $h$ to a bounded analytic function in the unit disc; also, each bounded analytic function arises as the Poisson integral of its boundary values

$$
h\left(e^{i \theta}\right)=\lim _{r \rightarrow 1} h\left(r e^{i \theta}\right)
$$

which exist almost everywhere. For a summary of these basic facts, see [6].

Lemma 6. Let $K$ be a closed subset of $X$, the maximal ideal space of $L^{\infty}$, and suppose the interior of $K$ is empty. If $u$ is any nonnegative continuous function on $K$, there exists a function $h$ in $H^{\infty}$ such that the modulus of $\hat{h}$ agrees with $u$ on $K$.

Proof. Let $m$ be the measure on $X$ which corresponds to normalized Lebesgue measure on the unit circle. In other words, if $\psi$ is the linear functional

$$
\psi(f)=\frac{1}{2 \pi} \int_{-\pi}^{\pi} f\left(e^{i \theta}\right) d \theta
$$

on $L^{\infty}$, the Riesz representation theorem, together with the isomorphism between $L^{\infty}$ and $C(X)$, provides us with a unique probability measure $m$ on $X$ such that

$$
\psi(f)=\int_{x} \hat{f} d m, \quad f \in L^{\infty} .
$$

Then $X$ is a hyperstonian space and $m$ is a normal measure on $X$, i.e., if $S$ is a measurable subset of $X, m$ assigns the same measure to $S$ and the interior of $S$ :

$$
m(S)=m(\operatorname{int} S)
$$

Consider the given closed set $K$ in $X$. Since $K$ has no interior, $m(K)=0$. Hence, we can construct a nonnegative continuous function on $X$ which vanishes on $K$ and has its logarithm integrable with respect to $m$. That is, there exists an $f$ in $L^{\infty}$ such that 
(a) $f \geqq 0$

(b) $\hat{f}=0$ on $K$

(c) $\int \log f\left(e^{i \theta}\right) d \theta>-\infty$.

Let $u$ be a nonnegative continuous function on $K$. We can extend $u$ to a nonnegative continuous function on $X$. Thus, we have a function $g$ in $L^{\infty}$ such that $g \geqq 0$ and $\hat{g}=u$ on $K$. With the function $f$ of the previous paragraph define

$$
h(z)=\exp \frac{1}{2 \pi} \int_{-\pi}^{\pi} \frac{e^{i \theta}+z}{e^{i \theta}-z} \log \left[f\left(e^{i \theta}\right)+g\left(e^{i \theta}\right)\right] d \theta .
$$

Since $\log (f+g)$ is integrable and bounded above, $h$ is a bounded analytic function in the unit disc. The boundary values

$$
h\left(e^{i \theta}\right)=\lim _{r \rightarrow 1} h\left(r e^{i \theta}\right)
$$

then satisfy

$$
|h|=f+g \text {, almost everywhere . }
$$

So $|\hat{h}|=\hat{f}+\hat{g}$. In particular, since $\hat{f}=0$ on $K$, we have

$$
|\hat{h}|_{K}=|\hat{g}|_{K}=u \text {. }
$$

The closed set $K$ of the last lemma may be sufficiently small so that the restriction of $\hat{H}^{\infty}$ to $K$ is all of $C(K)$. If $K$ is a nonempty $G_{\delta}$, that does not happen; however, we shall not stop to prove that here. We shall choose a particular $K$ on which it does not happen.

Let $K$ be the set of maximal ideals of $L^{\infty}$ which contain the function $(1-z)$. Then $K$ is a closed $G_{\delta}$ without interior in $X$, and the restriction of $\hat{H}^{\infty}$ to $K$ is a proper closed subalgebra of $C(K)$. See [6; page 187].

Let $\mathscr{B}$ be the Boolean algebra of measurable sets on the circle, modulo null sets. The Stone space of $\mathscr{B}$ is the space $X$. The Stone representation for $\mathscr{B}$ is simply the restriction of the Gelfand representation of $L^{\infty}$ to the collection of characteristic functions of measurable sets. According to Proposition 2, there exists a homeomorphism $\tau$, of $X$ into the space $\beta N-N$. We use $\tau$ and the set $K$ to define an algebra of continuous functions on $\beta N$. Let $A$ be the algebra of continuous functions $f$ on $\beta N$ such that, on the set $K$, the composition $f \circ \tau$ agrees with the restriction of a function in $\hat{H}^{\infty}$. We assert that

(i) $A$ is a uniformly closed subalgebra of $C(\beta N)$;

(ii) every nonnegative continuous function on $\beta N$ is the modulus of a function in $A$;

(iii) $A \neq C(\beta N)$.

It is clear that (i) and (iii) are satisfied. Property (ii) follows from this observation. Suppose $g$ is a nonnegative continuous function on 
a totally disconnected compact Hausdorff space $Y$. Let $M$ be a closed subset of $Y$, and let $h$ be a continuous complex-valued function on $Y$ such that the modulus of $h$ agrees with $g$ on $M$. Then, there exists $f$ in $C(Y)$ such that $\left.f\right|_{M I}=\left.h\right|_{M}$ and $|f|=g$ on all of $Y$.

\section{BIBLIOGRAPHY}

1. W. Badé, and P. C. Curtis, The Wedderburn decomposition of commutative Banach algebras, Amer. J. Math. 82 (1960), 851-866.

2. L. Carleson, An interpolation problem for bounded analytic functions, Amer. J. Math. 80 (1958), 921-930.

3. J. Dixmier, Sur certains espaces consideres par M. H. Stone, Summa Bras. Math. 11 (1951), 151-182.

4. L. Gilman, and M. Jerison, Rings of continuous functions, D. van Nostrand Company, Inc., Princeton, 1960.

5. W. Hayman, Interpolation by bounded functions, Ann. de l'Inst. Fourier XIII (1958), $277-290$.

6. K. Hoffman, Banach spaces of analytic functions, Prentice-Hall, Inc., Englewood Cliffs, 1962.

7. D. J. Newman, Interpolation in $H^{\infty}$, Trans. Amer. Math. Soc. 92 (1959), 501-507.

8. Sikorski, A theorem on extension of homomorphisms, Ann. Polon. Math. 21 (1948), $332-335$.

Massachusetts Institute of TeChNology

AND

BRANDEIS UNIVERSITY 


\title{
PACIFIC JOURNAL OF MATHEMATICS
}

\author{
EDITORS
}

\author{
H. SAmelson \\ Stanford University \\ Stanford, California \\ R. M. Blumenthal \\ University of Washington \\ Seattle, Washington 98105
}

\author{
J. DugundjI \\ University of Southern California \\ Los Angeles, California 90007
}

*Richard Arens

University of California

Los Angeles, California 90024

\section{ASSOCIATE EDITORS}
E. F. BECKENBACH
B. H. NeUmanN
F. WOLF
K. YoSIDA

\section{SUPPORTING INSTITUTIONS}

\author{
UNIVERSITY OF BRITISH COLUMBIA \\ CALIFORNIA INSTITUTE OF TECHNOLOGY \\ UNIVERSITY OF CALIFORNIA \\ MONTANA STATE UNIVERSITY \\ UNIVERSITY OF NEVADA \\ NEW MEXICO STATE UNIVERSITY \\ OREGON STATE UNIVERSITY \\ UNIVERSITY OF OREGON \\ OSAKA UNIVERSITY \\ UNIVERSITY OF SOUTHERN CALIFORNIA
}

\author{
STANFORD UNIVERSITY \\ UNIVERSITY OF TOKYO \\ UNIVERSITY OF UTAH \\ WASHINGTON STATE UNIVERSITY \\ UNIVERSITY OF WASHINGTON \\ * * * * \\ AMERICAN MATHEMATICAL SOCIETY \\ CALIFORNIA RESEARCH CORPORATION \\ SPACE TECHNOLOGY LABORATORIES \\ NAVAL ORDNANCE TEST STATION
}

Mathematical papers intended for publication in the Pacific Journal of Mathematics should by typewritten (double spaced). The first paragraph or two must be capable of being used separately as a synopsis of the entire paper. It should not contain references to the bibliography. No separate author's resumé is required. Manuscripts may be sent to any one of the four editors. All other communications to the editors should be addressed to the managing editor, Richard Arens, at the University of California, Los Angeles, California 90024.

50 reprints per author of each article are furnished free of charge; additional copies may be obtained at cost in multiples of 50 .

The Pacific Journal of Mathematics is published quarterly, in March, June, September, and December. Effective with Volume 13 the price per volume (4 numbers) is $\$ 18.00$; single issues, $\$ 5.00$. Special price for current issues to individual faculty members of supporting institutions and to individual members of the American Mathematical Society: $\$ 8.00$ per volume; single issues $\$ 2.50$. Back numbers are available.

Subscriptions, orders for back numbers, and changes of address should be sent to Pacific Journal of Mathematics, 103 Highland Boulevard, Berkeley 8, California.

Printed at Kokusai Bunken Insatsusha (International Academic Printing Co., Ltd.), No. 6, 2-chome, Fujimi-cho, Chiyoda-ku, Tokyo, Japan.

PUBLISHED BY PACIFIC JOURNAL OF MATHEMATICS, A NON-PROFIT CORPORATION

The Supporting Institutions listed above contribute to the cost of publication of this Journal, but they are not owners or publishers and have no responsibility for its content or policies.

* Basil Gordon, Acting Managing Editor until February 1, 1966. 


\section{Pacific Journal of Mathematics}

\section{Vol. 15, No. $4 \quad$ December, 1965}

Robert James Blattner, Group extension representations and the structure space ........... 1101

Glen Eugene Bredon, On the continuous image of a singular chain complex .............. 1115

David Hilding Carlson, On real eigenvalues of complex matrices .................... 1119

Hsin Chu, Fixed points in a transformation group ............................. 1131

Howard Benton Curtis, Jr., The uniformizing function for certain simply connected Riemann

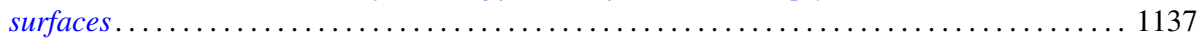

George Wesley Day, Free complete extensions of Boolean algebras................... 1145

Edward George Effros, The Borel space of von Neumann algebras on a separable Hilbert

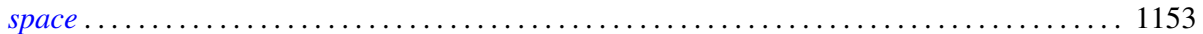

Michel Mendès France, $A$ set of nonnormal numbers ......................... 1165

Jack L. Goldberg, Polynomials orthogonal over a denumerable set ................ 1171

Frederick Paul Greenleaf, Norm decreasing homomorphisms of group algebras . . . . . . . . 1187

Fletcher Gross, The 2-length of a finite solvable group ........................ 1221

Kenneth Myron Hoffman and Arlan Bruce Ramsay, Algebras of bounded sequences ........ 1239

James Patrick Jans, Some aspects of torsion . . . . . . . . . . . . . . . . . . . . . . . 1249

Laura Ketchum Kodama, Boundary measures of analytic differentials and uniform

approximation on a Riemann surface ............................... 1261

Alan G. Konheim and Benjamin Weiss, Functions which operate on characteristic

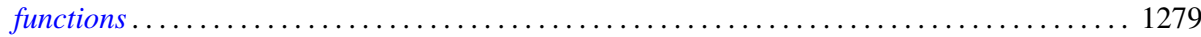

Ronald John Larsen, Almost invariant measures ............................ 1295

You-Feng Lin, Generalized character semigroups: The Schwarz decomposition ............ 1307

Justin Thomas Lloyd, Representations of lattice-ordered groups having a basis . . . . . . . . 1313

Thomas Graham McLaughlin, On relative coimmunity ....................... 1319

Mitsuru Nakai, $\Phi$-bounded harmonic functions and classification of Riemann surfaces ....... 1329

L. G. Novoa, On n-ordered sets and order completeness ..................... 1337

Fredos Papangelou, Some considerations on convergence in abelian lattice-groups . . . . . . . 1347

Frank Albert Raymond, Some remarks on the coefficients used in the theory of homology

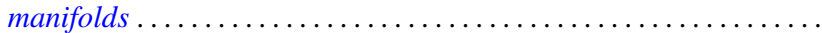

John R. Ringrose, On sub-algebras of a $C^{*}$-algebra .

Jack Max Robertson, Some topological properties of certain spaces of differentiable

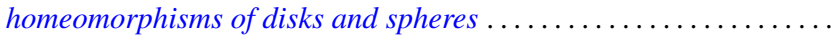

Zalman Rubinstein, Some results in the location of zeros of polynomials

Arthur Argyle Sagle, On simple algebras obtained from homogeneous general Lie triple systems. . . .

Hans Samelson, On small maps of manifolds ............................... 1401

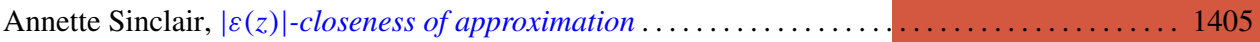

Edsel Ford Stiel, Isometric immersions of manifolds of nonnegative constant sectional curvature

Earl J. Taft, Invariant splitting in Jordan and alternative algebras ................. 1421

L. E. Ward, On a conjecture of R. J. Koch . . . . . . . . . . . . . . . . . . . . . . . . . . . 1429

Neil Marchand Wigley, Development of the mapping function at a corner . . . . . . . . . . 1435

Horace C. Wiser, Embedding a circle of trees in the plane ....................... 1463

Adil Mohamed Yaqub, Ring-logics and residue class rings . . . . . . . . . . . . . . . . 1465

John W. Lamperti and Patrick Colonel Suppes, Correction to: Chains of infinite order and their application to learning theory ........................................ 1471

Charles Vernon Coffman, Correction to: Non-linear differential equations on cones in Banach

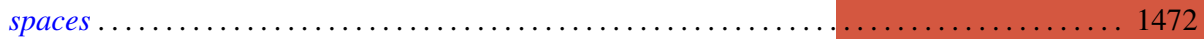

P. H. Doyle, III, Correction to: A sufficient condition that an $\operatorname{arc}$ in $S^{n}$ be cellular. . . . . . . . 1474

P. P. Saworotnow, Correction to: On continuity of multiplication in a complemented algebra 